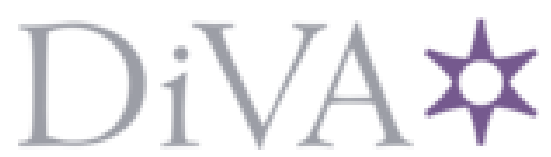

http://www.diva-portal.org

\title{
Postprint
}

This is the accepted version of a paper presented at 11th EAI International Conference on Heterogeneous Networking for Quality, Reliability, Security and Robustness (QSHINE 2015), Taipei, Taiwan, Aug. 19-20, 2015.

Citation for the original published paper:

Yu, R., Huang, X., Kang, J., Yuen, C., Vinel, A. et al. (2015)

An Optimal Replenishment Strategy in Energy Harvesting Wireless Networks with A Mobile Charger.

In: Der-Jiunn Deng, Lei Shu, Chih-Cheng Tseng, Chun-Cheng Lin, Neeli R. Prasad \& Tony Quek (ed.), 11th EAI International Conference on Heterogeneous Networking for Quality, Reliability,

Security and Robustness Taipei: Institute for Computer Sciences (ICST)

http://dx.doi.org/10.4108/eai.19-8-2015.2260685

N.B. When citing this work, cite the original published paper.

Permanent link to this version:

http://urn.kb.se/resolve?urn=urn:nbn:se:hh:diva-29194 


\section{An Optimal Replenishment Strategy in Energy Harvesting Wireless Networks with A Mobile Charger}

\author{
Rong Yu, Xumin Huang \\ and Jiawen Kang \\ Guangdong University of \\ Technology, China \\ Email: yurong@ieee.org, \\ huangxu.min@163.com, \\ Jiawen.kang.cn@ieee.org
}

\author{
Chau Yuen \\ Singapore University \\ of Technology and \\ Design, Singapore \\ Email: yuenchau@ sutd.edu.sg
}

\author{
Alexey Vinel \\ and Magnus Jonsson \\ Halmstad University, Sweden \\ Email: alexey.vinel@hh.se, \\ Magnus.Jonsson@hh.se
}

\author{
Stein Gjessing \\ and Yan Zhang \\ Simula Research Laboratory \\ and Department of Informatics \\ University of Oslo, Norway \\ Email: steing@ifi.uio.no, \\ yanzhang@ieee.org
}

\begin{abstract}
Energy harvesting wireless network is an emerging paradigm where the wireless nodes harvest energy to increase their lifetime. Due to high potential for a large variety of applications, such networks have drawn considerable attention in both industry and academia. In this paper, we consider the energy replenishment problem when the wireless nodes not only harvest energy from the ambient environment but also may receive energy from a mobile charger. We propose an optimal energy replenishment strategy using stochastic inventory theory to derive the optimal amount of energy supplied by the mobile charger. The simulation results show that our proposed scheme not only optimizes the energy utilization, but also improves the economic benefit of the wireless nodes.
\end{abstract}

\section{INTRODUCTION}

An energy harvesting wireless network consists of wireless nodes that are able to draw parts of their energy from the external environment by energy harvesting technologies [1], [2]. Recently, energy harvesting technologies have started to be applied in wireless sensor networks to extend the network lifetime and decrease maintenance costs [3]-[6]. However, longterm supply of energy in wireless sensor networks remains a challenging performance bottleneck. Harvested energy is often only available randomly, sporadically and in small amounts. Consequently, some wireless nodes may easily become energy deprived, calling for carefully designed energy management policies for data transmission. In addition, as the amount of data to be transmitted is increasing, the wireless nodes need more energy to be able to fulfill their transmission requirements [7].

Recent advances in wireless energy transfer justify the use of energy replenishment in energy harvesting networks. Energy replenishment schemes may utilize a mobile charger to prolong the lifetime and improve the efficiency of wireless networks [8]-[14]. A mobile charger can wirelessly transfer energy to wireless nodes with rechargeable batteries [9], [10]. Therefore, an energy harvesting wireless network with a mobile charger brings a new and promising direction to a large number of applications. The usage of mobile charger can be extended to most energy harvesting networks.

Several studies have utilized mobile chargers to prolong the lifetime of wireless sensor networks [8]-[14]. These studies usually consider energy replenishment and data flow routing in wireless nodes. A special node named Qi-ferry [9] is proposed to carry energy and wirelessly charge the wireless nodes. The authors solve a tradeoff problem of how many sensor nodes a Qi-ferry is able to charge, and how far the Qi-ferry must go back to recharge itself. In [8], a periodic charging scheme of a wireless charging vehicle is introduced. The authors propose an optimal traveling path to maximize the charging coverage of the wireless charging vehicle, and also formulate an optimization problem of data flow routing and energy recharging. Some practical constraints of the mobile charger are taken into consideration in [10], such as travel times and speed. In [11] and [12], the problem of scheduling multiple mobile chargers is studied to deal with the limited performance of only one mobile charger. Additionally, the scheme, that mobile chargers could not only charge the nodes but also carry their data, is investigated in [13], [14].

The aforementioned schemes have their limitations in practice, since they only work well under their specific assumptions and conditions. For instance, they may assume fixed generated data rates [8], [13], [14], the same amount of replenished energy [8], [9], [11], and the same size of energy storage for all nodes [11], [14]. The data rate of each node depends on the application running on the node and changes over time [10]. The amount of energy replenished from mobile chargers may be different for each node, but can be estimated from the demands of applications. In addition, it is not appropriate to assume that the energy in a battery is stored without any cost. Actually, the energy stored in a battery suffers with selfdischarging.

In this paper, we consider a much more practical scenario, in which there are energy storage cost in the nodes' batteries (e.g., self-discharging). Besides, the nodes should pay for the 
replenished energy from the mobile chargers. In this case, the wireless nodes should not replenish too much energy because of the energy storage cost and the payment to the mobile charger. However, if the nodes replenish insufficient energy, they will lose their chance to transmit data and earn economic benefit. By considering above practical scenarios, we are motivated to propose an optimal energy replenishment strategy for wireless nodes to work with mobile chargers in an energy harvesting wireless network. The wireless nodes can make an optimal decision using stochastic inventory theory to replenish optimal amount of energy from the mobile chargers. The simulation results indicate that our proposed scheme optimizes the energy utilization and also improves the economic benefit.

The main contributions of this paper can be summarized as follows.

- We derive a practical system model of energy harvesting wireless networks by considering three crucial conditions: the time-varying rate of generated data, the different amount of replenished energy for nodes and the cost of energy stored in batteries.

- We consider the energy replenishment problem through the framework of stochastic inventory theory, which is a new perspective for solving the problem of energy replenishment in energy harvesting wireless networks.

- We propose a strategy that not only optimizes energy utilization, but also improves the economic benefit of the wireless nodes. When requesting energy replenishment, the nodes consider the externally harvested energy to avoid replenishing and paying for excessive energy, which also optimizes battery energy utilization.

The rest of this paper is organized as follows. In Section II, we describe the system model including the energy consumption model of the nodes and the energy replenishment from the mobile charger. We formulate the optimal energy replenishment problem as an inventory problem in Section III. We present simulation results to evaluate the performance in Section IV. Finally, we draw the conclusion in Section V.

\section{System Model}

\section{A. Network model}

Fig. 1 shows an energy harvesting wireless network consisting of $N$ wireless nodes distributed over a two-dimensional area. The wireless nodes represent sensors, devices or machines, which may carry out sensing tasks and then transmit real-time sensing data to the base station (BS). Each wireless node has a rechargeable battery (or super-capacitor). The BS is deployed in a fixed location, and is a sink node for all nodegenerated data. Within the coverage area of the BS, all wireless nodes can directly transmit their data to the BS without relays. When node $i$ runs out of energy, it can replenish energy from a mobile charger instead of passively waiting for harvesting energy from the environment. The harvested energy is often available sporadically and in small amounts. Therefore, it is much more reasonable for wireless nodes to request energy replenishment from the mobile charger in most cases.

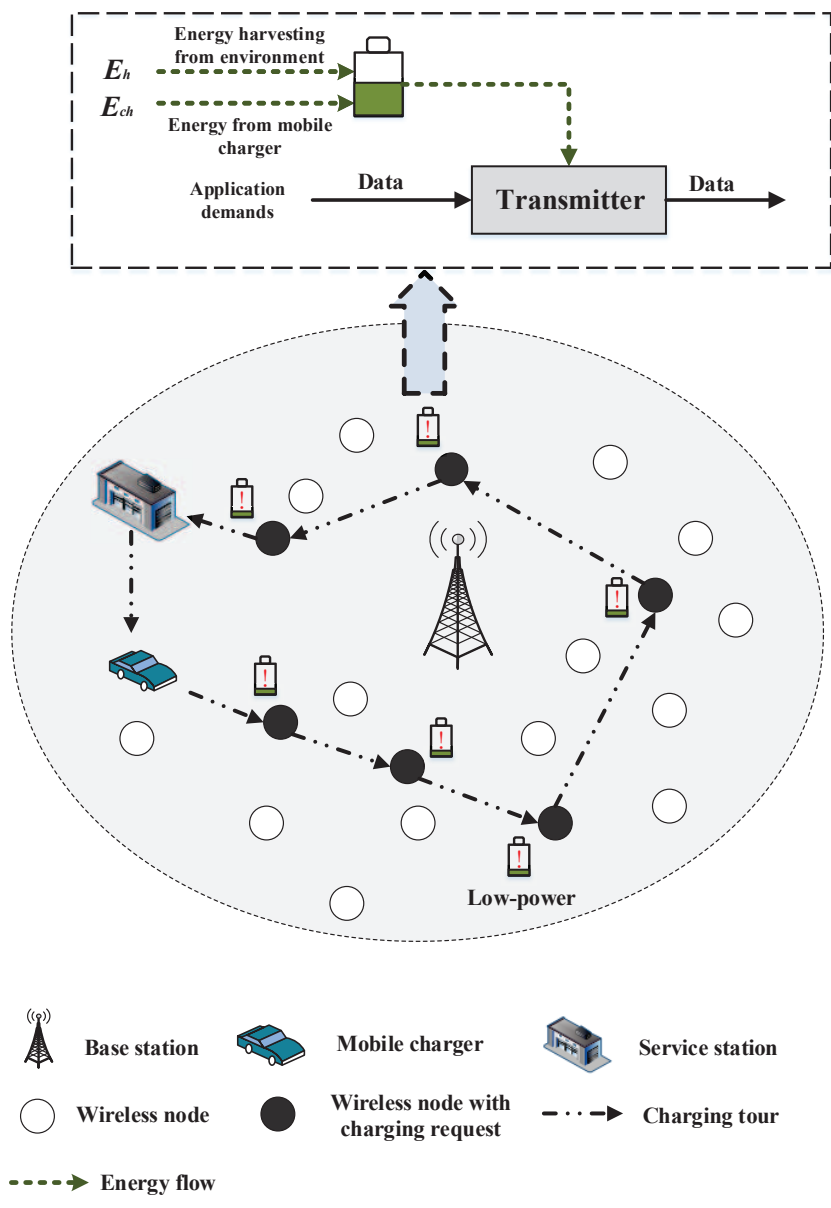

Fig. 1: A detailed example of an energy harvesting wireless network with a mobile charger.

A wireless charging vehicle works as a mobile charger to charge the nodes in the energy harvesting wireless network. The mobile charger keeps real-time communications with the BS to ensure information consistency [15]. Since the mobile charger is driven by petrol or electricity, it can travel with a constant speed and its traveling path is unconstrained. While traveling, the mobile charger can also receive requests via the BS. Compared with the travel time, the request delivery time and the charging time are negligible [11]. To maximize the energy wireless transfer efficiency, the mobile charger charges only one node each time. After finishing the point-to-point charging tasks, the mobile charger will return to the service station (its starting point) to recharge itself, and get ready for the next charging tour. A detailed example of the charging case is shown in Fig. 1, where wireless nodes will send requests to the BS based on their application demands and residual energy. The major notations used in this paper are listed in Table I.

\section{B. Dynamic energy consumption model}

The energy consumption rate of wireless nodes changes with applications and time. In this paper, the sensing data 
TABLE I: Notations and Descriptions

\begin{tabular}{c|l|c|l}
\hline Notations & Descriptions & Notations & Descriptions \\
\hline$E_{h, i}$ & energy harvested from environment of $i$ & $E_{i}$ & current residual energy in $i$ \\
$\bar{E}_{h, i}$ & average total amount of energy harvested from environment & $E_{r e, i}$ & energy replenished from mobile charger \\
$\lambda_{i}$ & the mean arrival rate of data & $e_{i}$ & energy consumption of $i$ per unit time \\
$D_{i}$ & distance between $i$ and the based station & $t_{r, i}$ & time interval between sending out request and receiving energy \\
$p_{r}$ & cost of energy replenishment from mobile charger & $p_{e}$ & the benefit from transmitting sensing data \\
$p_{l}$ & loss of missing transmitting sensing data & $p_{s}$ & storage cost of per energy unit in $i$ \\
$C_{i}$ & average total amount of transmitted data of $i$ & $L_{i}$ & average total amount of missing data of $i$ \\
$S_{i}$ & average total amount of storage in battery of $i$ & $P_{i}$ & the amount benefit of $i$ \\
\hline
\end{tabular}

arrival process of a wireless node $i$ follows a poisson process [16]. The mean arrival rate of data is $\lambda_{i}$ bps, which is more reasonable under most scenarios than that of many existing work, whose arrival rate of data is fixed and given. Similar to [12], each node can monitor its residual energy and estimate the demand of its applications. In other words, $\lambda_{i}$ can be estimated ahead of time according to historical records of applications.

As the size of request messages is very small, node $i$ mainly consumes its energy in transmitting data to the BS. According to [17], the energy consumption of node $i$ per unit time is denoted as

$$
e_{i}=\rho_{i} \lambda_{i}
$$

where $\rho_{i}$ is the energy consumption cost per unit data, which is given by

$$
\rho_{i}=\alpha_{1}+\alpha_{2} D_{i}^{m}
$$

where $\alpha_{1}$ and $\alpha_{2}$ are two given constants. $D_{i}$ is the distance between node $i$ and the BS, and $m$ is the loss index of the transmission path. In an energy harvesting wireless network, node $i$ can harvest the energy from the ambient environment, which is denoted by $E_{h, i}$. The process of energy harvesting is assumed to be stationary ergodic and mutually statistical independent between different wireless nodes. For the sake of simplicity, the process of energy harvesting is a sequence of independent identically distributed (iid) random variables, where the amount of energy harvested takes values from the set $\sigma=[0.25,0.5,0.75,1] \mu J$ with equal probability every time unit [18]. When the nodes run out of energy, they will apply for energy replenishment from a mobile charger. Let $E_{r e, i}$ be the energy supplied by the mobile charger to node $i$. Then node $i$ can be charged with the energy, $E_{c h, i}$, which satisfies

$$
E_{c h, i}=\beta E_{r e, i} .
$$

Here, $\beta$ is the efficiency of the wireless energy transfer [9].

\section{PRoblem Formulation AND SOlution}

In this section, we formulate the energy replenishment problem using stochastic inventory theory for an energy harvesting wireless network with a mobile charger. We also discuss a scenario to illustrate the optimal energy replenishment strategy.

\section{A. Energy replenishment problem formulation}

When node $i$ requests energy replenishment, the mobile charger will arrive at the location of node $i$ and then begin to charge it. The time interval between sending out a request and receiving energy, $t_{r, i}$, is called the time interval of replenishment for node $i$. The $t_{r, i}$ is a random variable modeling the task schedule of the mobile charger [10]-[12]. The distribution characteristics of the time interval of replenishments can be estimated from the historical records by statistical methods. We assume that $t_{r, i}$ takes value from $\left[t_{r, \min }, t_{r, \max }\right]$, and that the probability density function is given as $f\left(t_{r, i}\right)$. Because of the dynamic nature of the time intervals of replenishments $t_{r, i}$, node $i$ may run out of energy and be idle.

We consider that the cost of energy replenishment from a mobile charger is $p_{r}$ per unit energy. Meanwhile, a wireless node can earn some benefits by transmitting their sensing realtime data to the BS. The benefit is $p_{e}$ per unit of data. The data is required to be transmitted in time. When a wireless node runs out of energy, the wireless node will miss data transmission and suffer loss. The loss of a missing data transmission event is denoted $p_{l}$ per data unit. Moreover, the nodes in an energy harvesting wireless network typically utilize a super-capacitor to store energy nowadays. The energy storage cost can not be ignored as the super-capacitors are expensive in terms of cost per watt [19]. The cost of energy storage is $p_{s}$ per unit time.

During two consecutive energy replenishment time intervals, node $i$ can earn economic benefit from data transmissions. On one hand, it is unwise for node $i$ to request too much energy from the mobile charger each time, because node $i$ has to pay more. The energy storage cost is increased with the increasing energy stored in the battery or super-capacitor. On the other hand, if it requests only a small amount of energy, it should use more time to finish data transmissions and earn less. Hence, node $i$ should request a certain amount of energy, based on both predicted energy demand caused by data transmissions and on the dynamic nature of $t_{r, i}$.

As shown in Fig. 2, in an energy harvesting wireless network, every wireless node is like a salesman that has its own energy storage system (a battery or super-capacitor). The transmitting data is similar as products. Node $i$ not only harvests energy from the environment but also requests energy 
replenishment from the mobile charger (like a manufacturer) to transmit data. It is similar to the scenario that a salesman stores and sells his products to the customers (i.e., base station), and then earns from them. He also has to pay for the raw material from the manufacturer. The problem of maximizing the wireless nodes' economic benefit can be formulated into an inventory control problem. Here, the inventory control problem is similar as that of how much energy a salesman should order to satisfy the demand of his products in each time period. We utilize this optimal inventory approach to solve the energy storage problem. The application demands of the wireless nodes are stochastic. Similarly with the scenario in [20], each node should make an optimal decision to control its inventory of energy in order to maximize its economic benefit. The wireless nodes should determine the optimal amount of replenished energy from the mobile charger. By the productsinventory model of energy replenishment, an optimal energy replenishment strategy is then proposed to decide the amount of replenished energy.

\section{B. An optimal energy replenishment strategy using stochastic inventory theory}

In an energy harvesting wireless network, we define $\Delta t_{i}$ to be the energy consumption duration of node $i$ between the last time of battery replenshment from the mobile charger and when running out of energy. On average, if node $i$ consumes $e_{i}$ energy every time unit, the energy consumption time $\Delta t_{i}$ is given by

$$
\Delta t_{i}=\frac{E_{i}+E_{c h, i}+\bar{E}_{h, i}}{e_{i}}
$$

where $E_{i}$ is the current residual energy in the battery of node $i$. $\bar{E}_{h, i}$ is the average total amount of energy harvested from the environment within the energy consumption time $\Delta t_{i} . E_{c h, i}$ is the total charged energy from the wireless charging vehicles. As the energy harvested from ambient environment is taken from the set $\sigma=[0.25,0.5,0.75,1] \mu J$ with equal probability each time unit, the average total amount of harvested energy is $\bar{E}_{h, i}=0.625 \Delta t_{i}$. From eqn. (4), it is clear that the longer energy consumption time results from requesting more replenished energy. If the time interval of replenishment $t_{r, i}$ is larger than the energy consumption time $\Delta t_{i}$, node $i$ will be short of energy to transmit data and will be idle until it receives enough energy to work again.

Fig. 2 shows that it is important for a salesman to choose the optimal inventory level to satisfy the demand of sending products in an inventory system. The salesman should also avoid redundant inventory in each time period. The economic benefit of a node depends on the following factors: the amount of energy replenishment, the inventory in the wireless node, the amount of transmitted data and the loss of missing data transmission. Generally, when increasing the loss of missing data transmission or the replenished energy, the economic benefit of nodes will decrease. On the other hand, the economic benefit can be improved by increasing the amount of

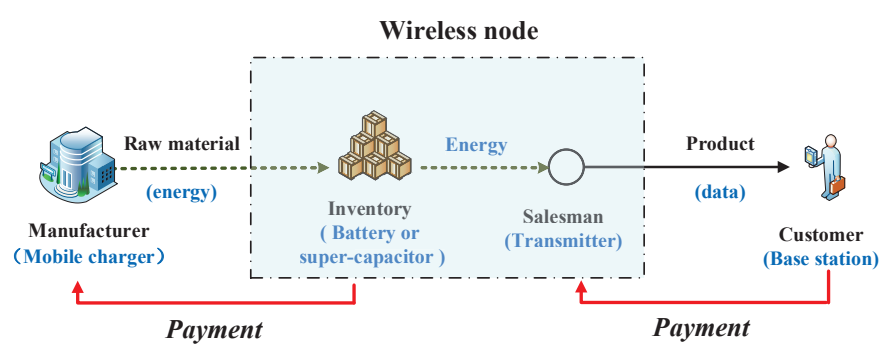

Fig. 2: The model of an optimal energy replenishment strategy using stochastic inventory theory.

transmitted data. We solve the optimal energy replenishment problem by using stochastic inventory theory.

In our life, if a salesman has insufficient inventory, he could not send products to his customers. Then his customers will choose to leave, which results in a loss for the salesman. Analogously, if node $i$ is short of energy, the event of missing data transmissions happens. The average total amount of missing data, $L_{i}$, is expressed as

$$
L_{i}=\int_{\Delta t_{i}}^{t_{r, \max }} f\left(t_{r, i}\right)\left(t_{r, i}-\Delta t_{i}\right) d t_{r, i}
$$

Apart from the missing data, the rest of the sensed data from a node can be successfully transmitted before running out of energy. The average total amount of transmitted data for node $i$ is given by

$$
C_{i}=\int_{t_{r, \text { min }}}^{\Delta t_{i}} \lambda_{i} t_{r, i} f\left(t_{r, i}\right) d t_{r, i}+\int_{\Delta t_{i}}^{t_{r, \max }} \lambda_{i} \Delta t_{i} f\left(t_{r, i}\right) d t_{r, i} .
$$

There are two items in eqn. (6). The first item is the average value of data transmission when $t_{r, i} \leq \Delta t_{i}$. The second item is the average value of data transmission when $t_{r, i}>\Delta t_{i}$.

To transmit the aforementioned data, the average total amount of energy is represented as

$$
R_{i}=\rho_{i} C_{i} .
$$

The cost of energy includes both the replenished energy and the current energy inventory. During each time period, the amount of stored energy is varying with the amount of replenished energy. Clearly, more replenished energy brings bigger energy inventory. Then, the total amount of storage in energy inventory, $S_{i}$, is represented by

$$
\begin{aligned}
& S_{i}=\rho_{i}\left(\int_{t_{r, \text { min }}}^{\Delta t_{i}} f\left(t_{r, i}\right) \int_{0}^{t_{r, i}} \lambda_{i}\left(\Delta t_{i}-t\right) d t d t_{r, i}+\right. \\
& \left.\int_{\Delta t_{i}}^{t_{r, \max }} f\left(t_{r, i}\right) \int_{0}^{\Delta t_{i}} \lambda_{i}\left(\Delta t_{i}-t\right) d t d t_{r, i}\right) .
\end{aligned}
$$

Given all the price parameters and known variables, the average total economic benefit of node $i$ in our energy inventory model, $P_{i}$, is expressed by,

$$
P_{i}=p_{e} C_{i}-p_{l} L_{i}-p_{s} S_{i}-p_{r} R_{i} .
$$


From eqn. (5), (6), (7) and (8), we know that eqn. (9) is a function of the variable $\Delta t_{i}$. To maximize the economic benefit, the optimal energy consumption time, $\Delta t_{i}^{*}$, is the key point. The objective function is defined as

$$
\Delta t_{i}^{*}=\underset{0 \leq \Delta t_{i} \leq t_{r}^{\max }}{\arg } \max \left(P_{i}\left(\Delta t_{i}\right)\right) .
$$

The optimal amount of requested energy for replenishment, $E_{r, i}^{*}$, can be found by deriving the above equations.

\section{A case study for the optimal inventory solution}

Similarly to the case in [12], we consider that the replenishment time interval $t_{r, i}$ follows a uniform distribution. The steps of an optimal inventory solution are as follows.

- Step 1: The probability density function $f\left(t_{r, i}\right)$ is denoted by,

$$
f\left(t_{r, i}\right)=\frac{1}{t_{r, \max }-t_{r, \min }}, t_{r, i} \in\left[t_{r, \min }, t_{r, \max }\right] .
$$

- Step 2: According to eqn. (11), the average total amount of transmitted data $C_{i}$, the total amount of stored energy $S_{i}$ and the total amount of missing data $L_{i}$ can be expressed as follows, respectively,

$$
\begin{gathered}
C_{i}=\frac{\lambda_{i}}{t_{r, \max }-t_{r, \min }}\left[\Delta t_{i} t_{r, \max }-0.5\left(\Delta t_{i}{ }^{2}+t_{r, \min }^{2}\right)\right] \\
S_{i}=\frac{\lambda_{i} \rho_{i}}{t_{r, \max }-t_{r, \min }}\left[\frac{\Delta t_{i}\left(t_{r, \max } \Delta t_{i}-t_{r, \min }^{2}\right)}{2}-\right. \\
\left.\frac{\Delta t_{i}{ }^{3}-t_{r, \min }^{3}}{6}\right] \\
L_{i}=\frac{0.5 \lambda_{i}}{t_{r, \max }-t_{r, \min }}\left(\Delta t_{i}-t_{r, \max }\right)^{2}
\end{gathered}
$$

- Step 3: To maximize the benefit, $P_{i}$, we can use the derivative method. Taking the first order partial derivative of $P_{i}$ with respect to $\Delta t_{i}$, we obtain

$$
\begin{aligned}
& \frac{\partial P_{i}}{\partial \Delta t_{i}}=\frac{\lambda_{i}}{t_{r, \max }-t_{r, \min }}\left[\left(p_{e}-\rho_{i} p_{r}+p_{l}\right)\left(t_{r, \max }-\Delta t_{i}\right)\right. \\
& \left.-\rho_{i} p_{s}\left(\Delta t_{i} t_{r, \max }-0.5 \Delta t_{i}^{2}\right)\right] .
\end{aligned}
$$

Then the second order partial derivative is given by,

$$
\begin{aligned}
& \frac{\partial^{2} P_{i}}{\partial \Delta t_{i}^{2}}=-\frac{\lambda_{i}}{t_{r, \text { max }}-t_{r, \text { min }}}\left[p_{e}-\rho_{i} p_{r}+p_{l}+\rho_{i} p_{s}\left(t_{r, \max }\right.\right. \\
& \left.\left.-\Delta t_{i}\right)\right] \\
& =-\frac{\lambda_{i}}{t_{r, \max }-t_{r, \min }} \Delta_{i} .
\end{aligned}
$$

Here, $\Delta_{i}=\left[p_{e}-\rho_{i} p_{r}+p_{l}+\rho_{i} p_{s}\left(t_{r, \max }-\Delta t_{i}\right)\right]$.

- Step 4: If $\Delta_{i}$ is smaller than 0 , we know that $P_{i}$ can be maximized, and $\Delta t_{i}^{*}$ can also be found by deriving $\frac{\partial P_{i}}{\partial \Delta t_{i}}=0$ in eqn. (15).

\section{Numerical RESUlts}

In this section, we evaluate the performance of the proposed optimal energy replenishment strategy in an energy harvesting wireless network by simulations.

We consider an energy harvesting wireless network consisting of 50 wireless nodes randomly deployed in a square area of $1 \times 1 \mathrm{~km}^{2}$. The base station is located in the middle

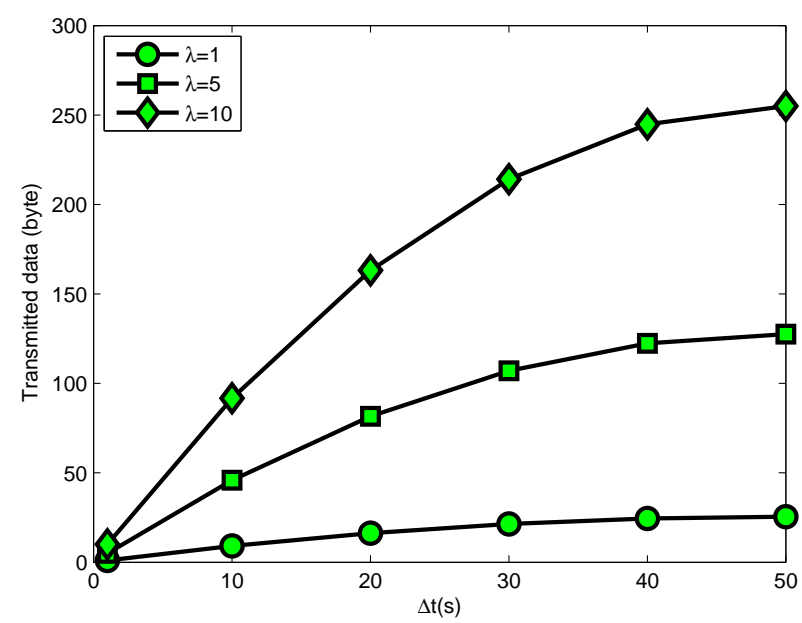

(a) The total amount of transmitted data.

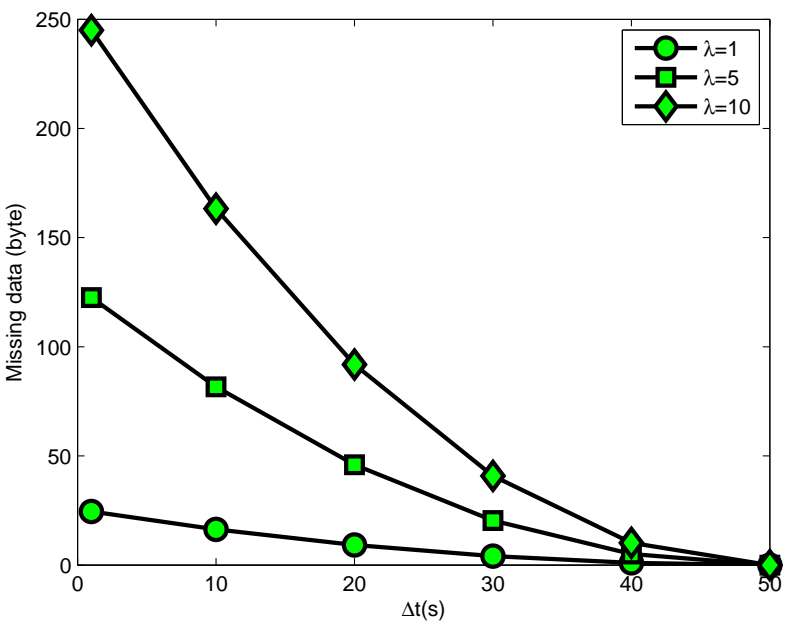

(b) The total amount of missing data.

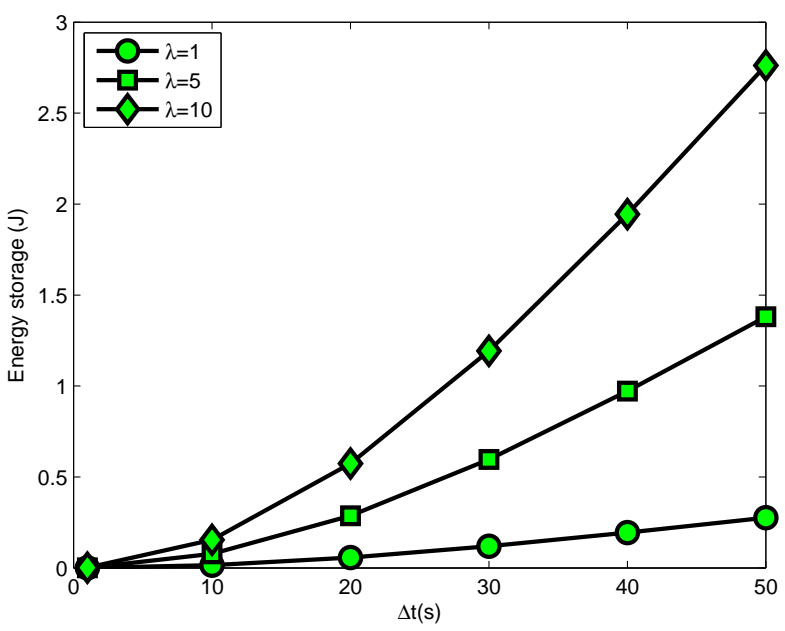

(c) The total amount of energy stored in the battery.

Fig. 3: A comparison of the total amount of transmitted data, missing data and energy storage (on average). 


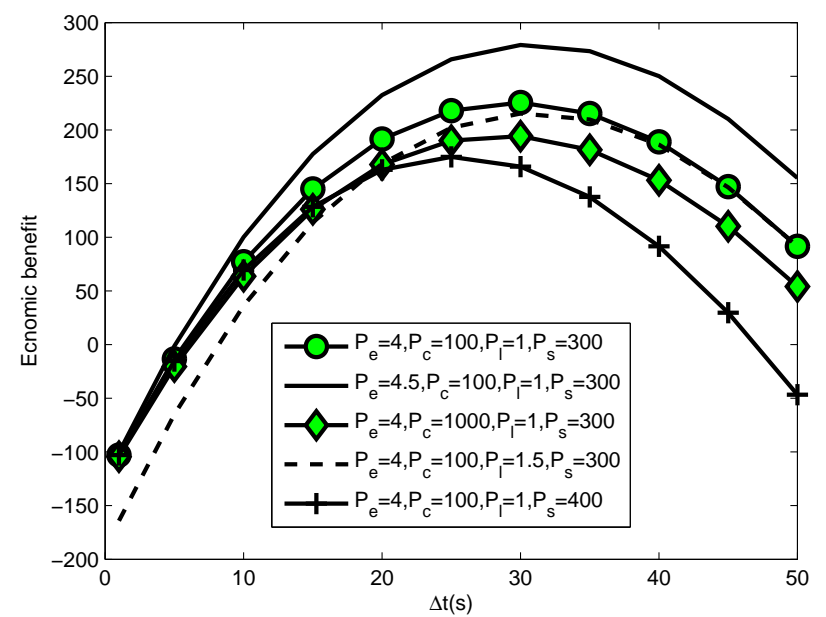

Fig. 4: The economic benefit of energy replenishment.

of this area. The energy consumption constants are set to $\alpha_{1}=50 \mathrm{~nJ} / \mathrm{b}$ and $\alpha_{2}=0.0013 \mathrm{pJ} /\left(\mathrm{b} \cdot \mathrm{m}^{4}\right)$. The loss index of the transmission path is $m=4$ [17]. The replenishment time interval is randomly chosen from the interval [1, 50].

Fig. 3 shows the transmitted data, missing data and energy storage during different energy consumption times $\Delta t$ for all nodes in an energy harvesting wireless network with a mobile charger. The lines with circles represent the case when the data arrival rate $\lambda=1$ bps. The lines with square represent $\lambda=5 \mathrm{bps}$ and the lines with diamond are $\lambda=10 \mathrm{bps}$. Fig. 3(a) shows the transmitted data during different $\Delta t$ s. It is clear that the longer energy consumption time $\Delta t$ brings the larger amount of transmitted data. Also, a larger data arrival rate results in a larger amount of transmitted data. Fig. 3(b) shows that the total amount of missing data is decreasing when $\Delta t$ increasing. Notice that the energy storage is increasing in the same scenario, showing that the nodes have more energy. In summary, when the energy consumption time increases (i.e., the nodes received more energy in the last energy replenishment), the energy storage in the batteries is larger. The wireless nodes have more energy to support data transmission, which results in more data transmitted. The amount of missing data will be less in this scenario.

Fig. 4 shows the average economic benefit of nodes with respect to the energy consumption time when the data arrival rate is $\lambda=5$ bps. From this figure, we know that the average economic benefit is also influenced by the following price parameters: $p_{r}, p_{s}, p_{l}$ and $p_{e}$. The $\Delta t^{*}$ increases with increasing $p_{e}$, and decreases when increasing the $p_{r}, p_{e}$ and $p_{s}$. The $\Delta t^{*}$ is decided by the pricing model, which brings the best economic benefit to nodes.

Fig. 5 shows the performance comparison between our optimal energy replenishment strategy and the maximal energy replenishment strategy. We evaluate the average economic benefit of 50 nodes with different data arrival rate during same time period. The horizontal axis represents the average data arrival rate of all the nodes. The vertical axis is the

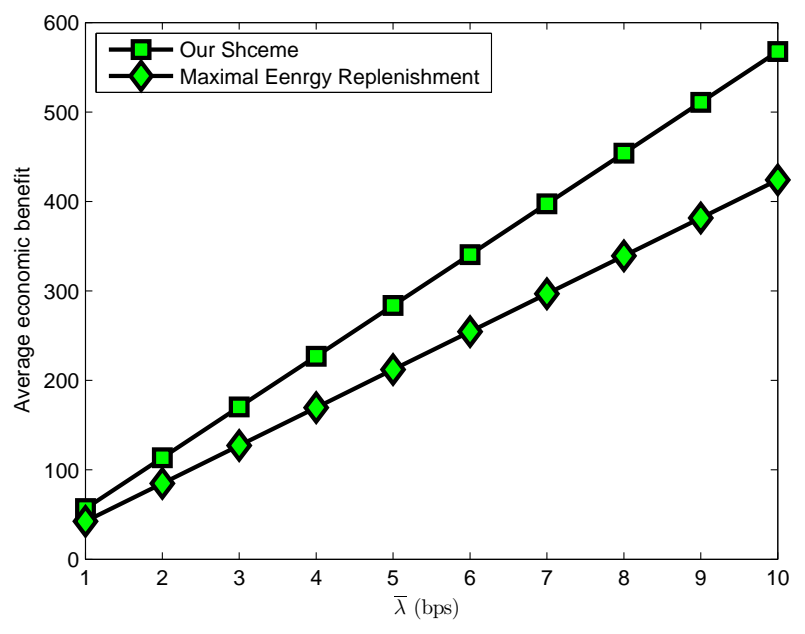

Fig. 5: The performance comparison of different strategies.

average economic benefit of all the nodes. In the maximal energy replenishment strategy, the wireless nodes replenish the maximal capacity of energy to their batteries or supercapacitors. Then, when the average data arrival rate increases, the average economic benefit of nodes is increased as well. Our optimal energy replenishment strategy is about 30\% higher than that of the maximal energy replenishment strategy when $\lambda=8$ bps. The reasons are as follows. Our scheme can optimize the total amount of energy replenishment to avoid high cost in energy storage. Additionally, the nodes also consider the external harvested energy from the environment to avoid replenishing too much energy from the mobile charger. In summary, our proposed strategy optimizes energy utilization, and also maximizes average economic benefit of wireless nodes.

\section{CONCLUSION}

In this paper, we consider optimal energy replenishment in energy harvesting wireless networks with a mobile charger. Stochastic inventory theory is used to maximize the economic benefit of the nodes. The proposed strategy allows the wireless nodes to make optimal decisions to replenish energy from the mobile chargers. As a consequence, a node can work collaboratively with the mobile charger. Simulation results indicate that the proposed strategy is efficient in maximizing both energy utilization and data transmission in energy harvesting wireless networks with a mobile charger.

\section{ACKNOWLEDGEMENT}

The work is supported in part by programs of NSFC under Grant nos. 61422201, 61370159 and U1201253, U1301255, Guangdong Province Natural Science Foundation under Grant no. S2011030002886, High Education Excellent Young Teacher Program of Guangdong Province under grant no. YQ2013057, and Science and Technology Program of Guangzhou under grant no. 2014J2200097 (Zhujiang New Star Program), 240079/F20 funded by the Research Council 
of Norway, the European Commission FP7 Project CROWN (grant no. PIRSES-GA-2013-627490), and Singapore A*Star SERC 1420200043.

\section{REFERENCES}

[1] S. Sudevalayam and P. Kulkarni, "Energy harvesting sensor nodes: Survey and implications," Communications Surveys \& Tutorials, IEEE, vol. 13 , no. 3, pp. 443-461, 2011.

[2] Y. K. Tan and S. K. Panda, "Review of energy harvesting technologies for sustainable wireless sensor network," Sustainable Wireless Sensor Networks, INTECH Publisher, pp. 15-43, 2010.

[3] U. Prathap, D. Shenoy, K. Venugopal, and L. Patnaik, "Wireless sensor networks applications and routing protocols: survey and research challenges," in International Proc. on Cloud and Services Computing (ISCOS), pp. 49-56, IEEE, 2012.

[4] A. M. Zungeru, L.-M. Ang, S. Prabaharan, and K. P. Seng, "Radio frequency energy harvesting and management for wireless sensor networks," Green Mobile Devices and Networks: Energy Optimization and Scavenging Techniques, pp. 341-368, 2012.

[5] S. Escolar, S. Chessa, and J. Carretero, "Optimization of quality of service in wireless sensor networks powered by solar cells," in IEEE 10th International Proc. on Parallel and Distributed Processing with Applications (ISPA), pp. 269-276, IEEE, 2012.

[6] W. Haboubi, H. Takhedmit, O. Picon, and L. Cirio, "A gsm-900 mhz and wifi-2.45 ghz dual-polarized, dual-frequency antenna dedicated to rf energy harvesting applications," in 7th European Proc. on Antennas and Propagation (EuCAP), pp. 3959-3961, IEEE, 2013.

[7] I. Jawhar, M. Ammar, S. Zhang, J. Wu, and N. Mohamed, "Ferrybased linear wireless sensor networks," in Proc. of the IEEE Global Telecommunications Conference (GLOBECOM), 2013.

[8] L. Xie, Y. Shi, Y. T. Hou, and H. D. Sherali, "Making sensor networks immortal: An energy-renewal approach with wireless power transfer," IEEE/ACM Transactions on Networking (TON), vol. 20, no. 6, pp. 17481761, 2012.

[9] K. Li, H. Luan, and C.-C. Shen, "Qi-ferry: Energy-constrained wireless charging in wireless sensor networks," in IEEE Wireless Communications and Networking Conference (WCNC), pp. 2515-2520, IEEE, 2012.

[10] X. Ren, W. Liang, and W. Xu, "Maximizing charging throughput in rechargeable sensor networks," in 23rd International Proc. on Computer Communication and Networks (ICCCN), pp. 1-8, IEEE, 2014.

[11] R. Beigel, J. Wu, and H. Zheng, "On optimal scheduling of multiple mobile chargers in wireless sensor networks," in Proceedings of the first international workshop on Mobile sensing, computing and communication, pp. 1-6, ACM, 2014.

[12] W. Xu, W. Liang, X. Lin, G. Mao, and X. Ren, "Towards perpetual sensor networks via deploying multiple mobile wireless chargers," in 43rd International Proc. on Parallel Processing (ICPP), pp. 80-89, IEEE, 2014.

[13] L. Xie, Y. Shi, Y. T. Hou, W. Lou, H. D. Sherali, and S. F. Midkiff, "Bundling mobile base station and wireless energy transfer: Modeling and optimization," in Proceedings IEEE INFOCOM, pp. 1636-1644, IEEE, 2013

[14] L. Xie, Y. Shi, Y. T. Hou, W. Lou, and H. D. Sherali, "On traveling path and related problems for a mobile station in a rechargeable sensor network," in Proc. of the fourteenth ACM international symposium on Mobile ad hoc networking and computing, pp. 109-118, ACM, 2013.

[15] Z. Li, Y. Peng, W. Zhang, and D. Qiao, "J-roc: a joint routing and charging scheme to prolong sensor network lifetime," in 19th IEEE International Proc. on Network Protocols (ICNP), pp. 373-382, IEEE, 2011

[16] S. Jain, R. C. Shah, W. Brunette, G. Borriello, and S. Roy, "Exploiting mobility for energy efficient data collection in wireless sensor networks," Mobile Networks and Applications, vol. 11, no. 3, pp. 327-339, 2006.

[17] Y. T. Hou, Y. Shi, and H. D. Sherali, "Rate allocation and network lifetime problems for wireless sensor networks," IEEE/ACM Transactions on Networking (TON), vol. 16, no. 2, pp. 321-334, 2008

[18] R. Rajesh, V. Sharma, and P. Viswanath, "Information capacity of energy harvesting sensor nodes," in IEEE International Proc. on Information Theory Proceedings (ISIT), pp. 2363-2367, IEEE, 2011

[19] http://batteryuniversity.com/learn/article/whats_the_role_of_the_ supercapacitor.

[20] E. L. Porteus, "Stochastic inventory theory," Handbooks in operations research and management science, vol. 2, pp. 605-652, 1990. 